\title{
Electrothermal flow in Dielectrophoresis of Single-Walled Carbon Nanotubes
}

\author{
Yuan $\operatorname{Lin}^{1}$, Junichiro Shiomi ${ }^{2}$, Shigeo Maruyama ${ }^{2}$, and Gustav Amberg ${ }^{1}$ 周 \\ 1: Department of Mechanics, Royal Institute of Technology, Osquare Backe 18, Stockholm, 100 44, Sweden \\ 2: Department of Mechanical Engineering, The University of Tokyo, \\ 7-3-1 Hongo, Bunkyo-ku, Tokyo, 113-8656, Japan
}

\begin{abstract}
We theoretically investigate the impact of the electrothermal flow on the dielectrophoretic separation of single-walled carbon nanotubes (SWNT). The electrothermal flow is observed to control the motions of semiconducting SWNTs in a sizeable domain near the electrodes under typical experimental conditions, therefore helping the dielectrophoretic force to attract semiconducting SWNTs in a broader range. Moreover, with the increase of the surfactant concentration, the electrothermal flow effect is enhanced, and with the change of frequency, the pattern of the electrothermal flow changes. It is shown that under some typical experimental conditions of dielectrophoresis separation of SWNTs, the electrothermal flow is a dominating factor in determining the motion of SWNTs.
\end{abstract}

PACS numbers: 47.65.-d,82.45.-h,73.63.Fg

\section{INTRODUCTION}

Single-walled carbon nanotubes are key materials in nanotechnology as potential candidates for diverse applications owning to their extraordinary mechanical, thermal, optical and electrical properties ${ }^{1}$. On exploring the utility of the electrical properties, one of the current critical challenges is the separation of metallic (m-SWNTs) and semiconducting SWNTs (s-SWNTs). Among various post-synthesis separation methods devised and applied 213/45/6, dielectrophoresis separation has been demonstrated to be possible with high selectivity and simplicity ${ }^{7}$. Furthermore, DEP also allows us to deposit SWNTs to selected sites to construct electric devices 8 . While the complexity in the optical measurements gives rise to difficulties in interpretation of the obtained spectra ${ }^{9}$, deeper understanding in the transport dynamics of SWNTs under DEP operation is of a great importance. It also allows us to discuss the efficiency and possibility for optimization of system designs and working conditions. Here, numerical simulations should serve as a powerful tool since the dynamical observation of SWNTs transported in the suspension is extremely challenging in experiments.

On modeling a SWNT as a prolate ellipsoid, the DEP force on the SWNT in an inhomogeneous AC electric field $\mathbf{E}$ is calculated as ${ }^{7}$,

$$
\mathbf{F}_{\mathrm{DEP}}=\frac{\pi a b^{2} \epsilon_{\mathrm{m}}}{12} \alpha \nabla|\mathbf{E}|^{2}
$$

where $\alpha=\operatorname{Re}\left[\frac{\epsilon_{p}^{*}-\epsilon_{\mathrm{m}}^{*}}{\epsilon_{\mathrm{m}}^{*}+\left(\epsilon_{p}^{*}-\epsilon_{\mathrm{m}}^{*}\right) L_{p}}\right], \quad \epsilon_{m, p}^{*}=\epsilon_{m, p}-i \frac{\sigma_{\mathrm{m}, \mathrm{p}}}{\omega}$, $\omega$ is the frequency of the AC field and $\epsilon_{\mathrm{m}, \mathrm{p}}^{*}$ is the complex dielectric permittivity. Here the subscripts $m$ and $p$ denote the medium and particle. The constants $a$ and $b$ denote half the tube-length and tube-radius. The depolarization factor $L_{p}=\frac{b^{2}}{2 a^{2} e^{3}}\left[\ln \left(\frac{1+e}{1-e}\right)-2 e\right], e=\sqrt{1-\frac{b^{2}}{a^{2}}}$. On calculating the DEP force of SWNTs using parameters from electrical measurements of suspended pure
SWNTs 10 , the force on m-SWNTs becomes several orders higher than that on s-SWNTs, indicating that the separation should be easy. Furthermore, numerical calculations suggested that DEP forces of both m-SWNTs and s-SWNTs are insensitive to variations in frequency of the AC electric field and surfactant concentration within the accessible range in experiments11. This does not agree with the experimental results where the success of DEP separation strongly depended on the frequency and the surfactant concentration ${ }^{7}$. Experiments showed that the separation was successful only above some certain frequencies below which both m-SWNTs and s-SWNTs were found on the electrodes. Although the complete picture of the transport dynamics is yet to be revealed, Krupke et $a^{[\pi}$ attributed the discrepancy to the electrical conduction through the surrounding surfactant. They have represented the surface conduction effect with the effective electrical conductivity which was obtained by fitting the solution of Eq. (1) to the experimental results.

Previous numerical simulations on DEP separation of SWNTs have usually considered only the DEP forces and the Brownian motion assuming that the bulk flow velocity is zero. Although the basic concept of DEPseparation in principle is simple, the system involves effects which may cause bulk flow motions such as electroosmosis, thermal convection, electrothermal flow. The electroosmosis effect is only important at low frequencies (less than $10^{4} \mathrm{~Hz}$, which is well below the frequency applied in experiments) $\frac{\sqrt{12}}{12}$, and the thermal convection is expected to be negligible in micro and nanoscales. On the other hand, the electrothermal effect is known to be substantial in micro systems ${ }^{12 \mid 13}$. Electrothermal flow is driven by a body force caused by electric field acting on gradients in permittivity and/or conductivity due to a non-uniform temperature field 12 .

In this paper, we investigate the impact of the electrothermal effect on the DEP-separation of SWNTs by formulating a dynamical model of the integrated system. Here we mainly discuss the transport of s-SWMT, to which electrothermal force has non-trivial effects. We demonstrate that for a commonly used surfactant and 
electric field with practical magnitude, electrothermal flows can be sufficiently large to have a substantial impact on the separation efficiency of the DEP method. It is shown that electrothermal flows can significantly weaken the DEP-separation by driving the s-SWNTs towards the electrodes, which is consistent with the experimental observations.

\section{MATHEMATICAL MODELING}

Using data for a commonly used material in experiments 7 , the system under consideration consists of SWNTs with a diameter of $1.4 \mathrm{~nm}$ and a length of 1 $\mu \mathrm{m}$ dispersed in aqueous solutions of sodium dodecylbenzene sulfonate (SDBS). For the electrical conductivity, we adopt the effective conductivity $(0.35 \mathrm{~S} / \mathrm{m})$ suggested by Krupke et $a^{\eta}$. While the influence of surfactant on the electrical conduction of SWNTs surrounded by SDBS is not clear, we adopt the empirically effective value. Similarly, the permittivity of s-SWNTs was set to be $5 \epsilon_{0} 7$. Although the magnitude of permittivity of s-SWNTs is arguable, a variation within one order of magnitude should not affect the current analysis.

Electrical conductivity of SDBS solutions $\sigma_{\mathrm{m}}$ was estimated as $4 \mathrm{mS} / \mathrm{m}, 29 \mathrm{mS} / \mathrm{m}$ and $230 \mathrm{mS} / \mathrm{m}$ for concentration of $0.01 \%, 0.1 \%$ and $1 \%$, respectively 7 . The permittivity of the SDBS solutions $\epsilon_{\mathrm{m}}$ at room temperature is about $80 \epsilon_{0}$ and has negligible dependence on the concentration. It is straightforward to calculate the DEP force factor for s-SWNTs and the results show that it decreases about one order when the concentration of SDBS increases by one order.

We solve the heat conduction equation with a heat source due to an electric field $\frac{13}{,}, k \nabla^{2} T+\sigma_{\mathrm{m}} E^{2}=0$, where the thermal conductivity $k=0.6 \mathrm{Wm}^{-1} \mathrm{~K}^{-1}$. As a typical system geometry in experiments 7 , a pair of 20 $\mu \mathrm{m}$-long and $1 \mu \mathrm{m}$-wide electrodes were located with a distance of $10 \mu \mathrm{m}$ at the bottom of the calculation domain as shown in Fig. 1(a), The boundary conditions are: $T_{\text {wall }}=300 \mathrm{~K}$ at the surrounding walls, and $\frac{d T}{d z}=0$ at the bottom, which means the substrate and electrodes are thermally insulated. Figure 1(b) shows the temperature profiles for various SDBS concentrations above the tip of the electrode $(x, y)=(0 \mu \mathrm{m}, 5 \mu \mathrm{m})$, with the applied $\mathrm{AC}$ potential $\phi=20 \mathrm{~V}$ (peak to peak). Maximum temperature differences are $0.3 \mathrm{~K}, 1.6 \mathrm{~K}$ and $11.8 \mathrm{~K}$ on the electrode $(\mathrm{z}=0)$ for $0.01 \%, 0.1 \%$ and $1 \%$ SDBS solutions, respectively.

The electrothermal body force acting on the bulk fluid due to the AC electric field is expressed as 13

$$
\mathbf{f}=-\frac{1}{2}\left[\left(\frac{\nabla \sigma_{\mathrm{m}}}{\sigma_{\mathrm{m}}}-\frac{\nabla \epsilon_{\mathrm{m}}}{\epsilon_{\mathrm{m}}}\right) \cdot \mathbf{E} \frac{\epsilon_{\mathrm{m}} \mathbf{E}}{1+(\omega \tau)^{2}}+\frac{1}{2}|\mathbf{E}|^{2} \nabla \epsilon_{\mathrm{m}}\right],
$$

where $\tau=\epsilon_{\mathrm{m}} / \sigma_{\mathrm{m}}$ is the charge relaxation time of the solution. The first term on the right hand side expresses the Coulomb force and the second the dielectric force.

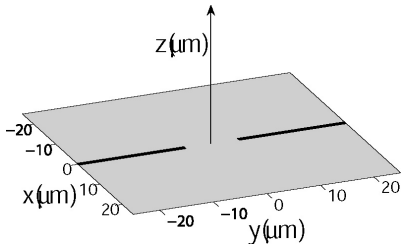

(a) Geometry of electrodes used in calculations

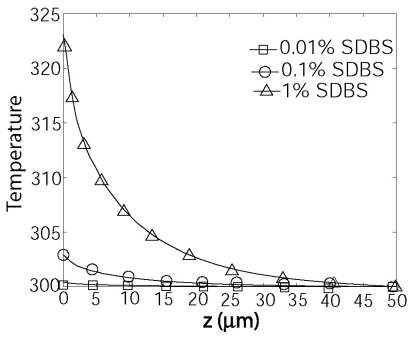

(b) Temperature changes with $z$ at $(x, y)=(0 \mu \mathrm{m}, 5 \mu \mathrm{m}), \phi$ is 20 $\mathrm{V}, \omega=300 \mathrm{kHz}$
FIG. 1: Geometry and temperature profiles.

For a typical electrolyte solution, $\left(1 / \sigma_{\mathrm{m}}\right) /\left(\partial \sigma_{\mathrm{m}} / \partial T\right)=$ $0.02 \mathrm{~K}^{-1}$ and $\left(1 / \epsilon_{\mathrm{m}}\right) /\left(\partial \epsilon_{\mathrm{m}} / \partial T\right)=-0.004 \mathrm{~K}^{-112}$. The first (second) term is dominant in the low (high) frequency regime (the crossover frequency is $\omega_{c}=1 / \tau$, which is $5.6 \mathrm{MHz}$ and $41 \mathrm{MHz}$ for $0.01 \%$ and $0.1 \%$ SDBS concentrations). In the calculation, we adopted $300 \mathrm{kHz}$ and $1 \mathrm{GHz}$, which represent the low and high frequency regime, respectively. For low Reynolds numbers, the transport term of the Navier-Stokes (N-S) equations can be neglected. Hence, the N-S equations become

$$
\rho_{m} \frac{\partial \mathbf{u}_{\mathrm{f}}}{\partial t}=\eta \nabla^{2} \mathbf{u}_{\mathrm{f}}-\nabla p+\mathbf{f} \quad \nabla \cdot \mathbf{u}_{\mathrm{f}}=0
$$

For low Reynolds numbers, the inertial effect can be neglected 12 . The terminal velocity of s-SWNTs is calculated as $\mathbf{u}=\mathbf{u}_{\mathrm{d}}+\mathbf{u}_{\mathrm{f}}+\mathbf{u}_{\mathrm{b}}$, here $\mathbf{u}_{\mathrm{d}}=\mathbf{F}_{\mathrm{DEP}} / f_{\mathrm{t}}$ with the translational friction coefficient for a prolate ellipsoid given by $f_{\mathrm{t}}=\frac{6 \pi \eta a}{\ln (2 a / b)}$. Characteristic variables of electrothermal flow are $U^{*}=\frac{\partial \sigma_{\mathrm{m}}}{\partial T}$. $\frac{25 \epsilon_{\mathrm{m}} \phi^{4}}{k\left(1+(\omega \tau)^{2}\right) \eta L}, T^{*}=\frac{\sigma_{\mathrm{m}} \phi^{2}}{k}, L=1 \mu \mathrm{m}$ and $t^{*}=\frac{\partial T}{\partial \sigma_{\mathrm{m}}}$. $\frac{k\left(1+(\omega \tau)^{2}\right) \eta}{25 \epsilon_{\mathrm{m}} \phi^{4}}$. Here $\phi$ is the applied peak-to-peak AC voltage. From the Einstein relation, the Brownian velocity can be derived as $\mathbf{u}_{b}=\sqrt{6 D / d t}$ with the diffusion coefficient $D=\left(k_{B} T / f_{\mathrm{t}}\right)$, and $d t$ is the time interval of observation. We define dimensionless variables $\tilde{\mathbf{u}}=\mathbf{u} / U^{*}, \tilde{T}=\left(T-T_{\text {wall }}\right) / T^{*}, \tilde{x}=x / L, \tilde{d t}=$ $d t / t^{*}$ and $\tilde{\mathbf{E}}=\mathbf{E} L / \phi$, dimensionless parameters $P_{1}=$ $\frac{\left(1+(\omega \tau)^{2}\right)}{12}, P_{2}=0.0463 \frac{\partial T}{\partial \sigma_{\mathrm{m}}} \frac{b^{2} \alpha \ln \left(\frac{a}{b}\right) k\left(1+(\omega \tau)^{2}\right)}{L^{2} \phi^{2}}$ and $P_{3}=\sqrt{\frac{\partial T}{\partial \sigma_{\mathrm{m}}} \frac{k_{B} \sigma_{\mathrm{m}} \ln \left(\frac{2 a}{b}\right) \cdot\left(1+(\omega \tau)^{2}\right)}{0.6 \cdot \epsilon_{\mathrm{m}} \phi^{2} \pi a}}$. Consequently, we obtain a set of system equations in a non-dimensional 
form,

$$
\begin{aligned}
\nabla \cdot \tilde{\mathbf{E}} & =0, \quad \nabla^{2} \tilde{T}=-\left|\tilde{\mathbf{E}}^{2}\right|, \\
\frac{\partial \tilde{\mathbf{u}}_{\mathrm{f}}}{\partial \tilde{t}} & =\nabla^{2} \tilde{\mathbf{u}}_{\mathrm{f}}-\nabla \tilde{p}+(\nabla \tilde{T} \cdot \tilde{\mathbf{E}}) \tilde{E}+P_{1}\left|\tilde{\mathbf{E}}^{2}\right| \nabla \tilde{T}, \\
\tilde{\mathbf{u}}_{\mathbf{b}} & =\tilde{r} \sqrt{\left(\tilde{T}+T_{\text {wall }} / T^{*}\right) / \tilde{d} t}, \quad \tilde{\mathbf{u}}_{\mathrm{d}}=\nabla|\tilde{\mathbf{E}}|^{2} \\
\tilde{\mathbf{u}} & =P_{2} \tilde{\mathbf{u}}_{\mathrm{d}}+\tilde{\mathbf{u}}_{\mathrm{f}}+P_{3} \tilde{\mathbf{u}}_{\mathrm{b}},
\end{aligned}
$$

where $\tilde{r}$ denotes a random number with normal distribution whose mean is 0 and standard deviation is 1. Equation (4) is solved using finite element method toolbox femLegd 14 .

\section{FORCE ESTIMATIONS}

Since the inertial effect is neglected, the dimensionless velocities are equivalent to the dimensionless forces. Taking the characteristic timescale $t^{*}$ as the time interval $d t$, the dependence of the relative magnitudes of DEP, Brownian and electrothermal forces on the applied electric potential was examined. Figure 2 shows the dimensionless forces at $(x, y, z)=(0 \mu \mathrm{m}, 14 \mu \mathrm{m}, 1 \mu \mathrm{m})$ for two different surfactant concentrations $0.01 \%$ and $0.1 \%$. The $y$ position was chosen to be in the regime with the positive electrothermal force that attracts SWNTs towards the electrode and $z=1 \mu \mathrm{m}$ equals to the length of SWNTs. Here, the frequency is $300 \mathrm{kHz}$, which is within the frequency range commonly used in experiments 78 . Figure 2 shows a clear dependence of the locally dominant force on the applied voltage and surfactant concentration. The change of the Brownian motion due to voltage and surfactant concentration is subtle and relatively negligible. The voltage-dependence of the strength of the electrothermal force is proportional to $\phi^{4}$ and that of the DEP force is proportional to $\phi^{2}$. As a result, for both concentrations $0.01 \%$ and $0.1 \%$, the electrothermal force overcomes the DEP forces at the high voltage limit since the electrothermal force increases with the voltage much faster than the DEP force.

As for the influence of the surfactant concentration, the electrothermal force increases with the concentration while the DEP force takes the opposite trend. When surfactant concentration is $0.01 \%$, the dominant force is the Brownian force for low voltage $(\phi<6 \mathrm{~V})$, the DEP force for intermediate voltage $(6 \mathrm{~V}<\phi<19 \mathrm{~V})$ and the electrothermal force for high voltage $(\phi>19 \mathrm{~V})$. On the other hand, when the concentration is $0.1 \%$, the local $\mathrm{DEP}$ force around the selected location is never dominant for the entire range of $\phi$. This implies that for SDBS concentration higher than $0.1 \%$, the positive DEP force on s-SWNTs plays minor role and the transport is governed mainly by the electrothermal force. The result suggests that there is a crossover between the electrothermal and DEP force with respect to the SDBS concentration. This means that beyond the crossover, an attempt to enhance the DEP-separation efficiency by increasing the surfactant concentration, i.e. by reducing the magnitude of

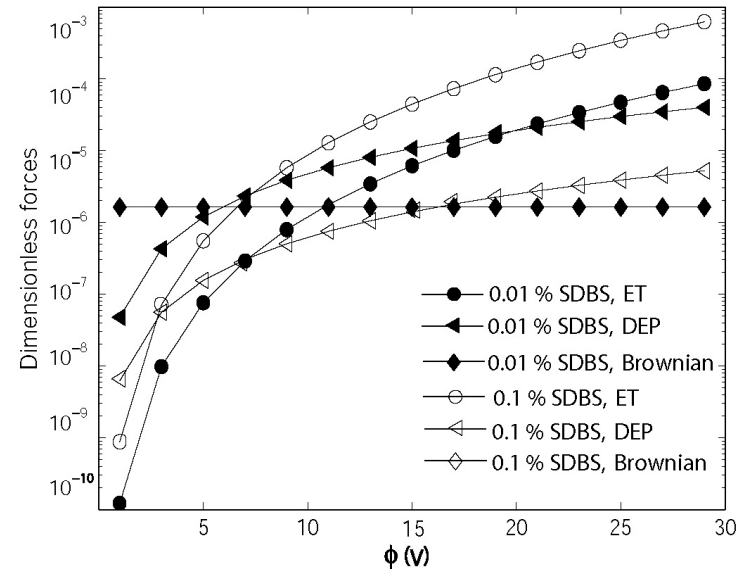

FIG. 2: Forces change with $\phi$ at $(x, y, z)=(0 \mu \mathrm{m}, 14 \mu \mathrm{m}$, $1 \mu \mathrm{m})$. The Brownian force profiles overlap on each other

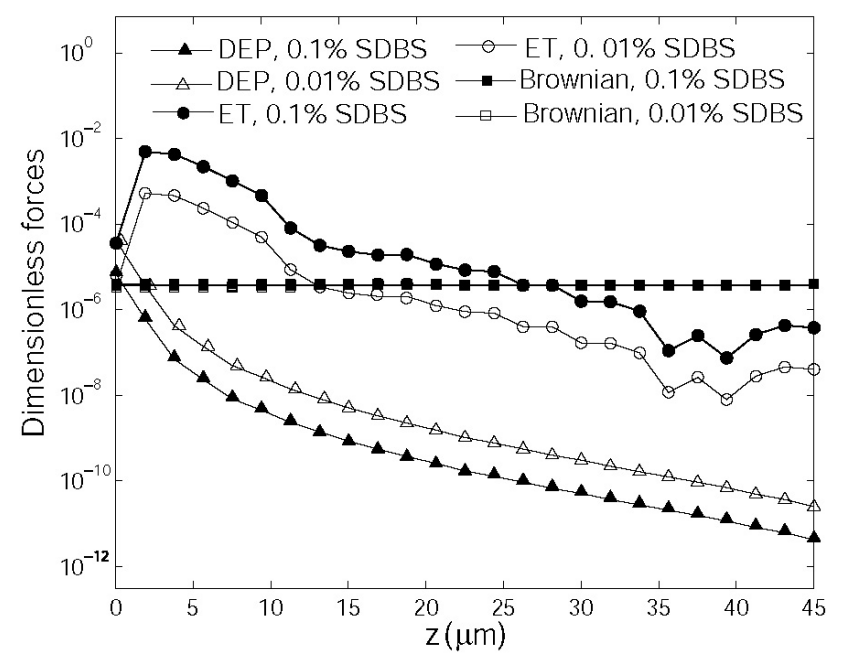

FIG. 3: Forces change with $\mathrm{z},(x, y)=(0 \mu \mathrm{m}, 14 \mu \mathrm{m}), \phi=20 \mathrm{~V}$. The Brownian force profiles overlap on each other

the positive DEP on s-SWNTs may result in enhancing the electrothermal force, which reduces the efficiency.

Figure 3 shows the magnitudes of the dimensionless forces with the vertical coordinate $z$ for the same $(x, y)$ locations as in Fig. 2. Following typical experiments, $\phi=20 \mathrm{~V}$ was applied to $0.1 \%$ and $0.01 \%$ SDBS solutions. Figure 3 shows that in $0.1 \%$ SDBS, the Brownian motion surpasses the DEP force except very close to the electrode (less than $1 \mu \mathrm{m}$ ). Here we observe that, in a domain larger than that where the DEP force is dominant, s-SWNTs are carried by the electrothermal flow for both SDBS concentrations. As a result, the electrothermal flow overcomes the Brownian motion in a relatively large domain and transport s-SWNTs onto the electrodes. To demonstrate this, we will verify the direction of the electrothermal flow and the actual traces of s-SWNTs under the action of the resultant force. 


\section{TRANSPORT SIMULATIONS OF SEMICONDUCTING SWNTS}

Figures 4(a) and 4(b) show streamlines of the electrothermal flows in 0.1\% SDBS solution for two different frequencies. They are cross-sectional views of the 3D simulation results at the plane of $x=0$. The thick line drawn from $(x, y)=(0 \mu \mathrm{m}, 5 \mu \mathrm{m})$ to $(0 \mu \mathrm{m}, 25 \mu \mathrm{m})$ marks one of the two electrodes [Fig. 1(a)]. The evident dependence of the flow pattern on $\omega$ is highlighted by comparing the flow pattern for $\omega=300 \mathrm{kHz}$ [Fig. 4(a)] corresponding to a typical working frequency with that for $\omega=1 \mathrm{GHz}$ [Fig. 4(b)] corresponding to the upper limit of frequency explored in experiments. When $\omega=300 \mathrm{kHz}$, the vortex core appears close to the gap of the electrodes, whereas when $\omega=1 \mathrm{GHz}$, it is located close the domain boundary $(y=25 \mu \mathrm{m})$. The pattern of the electrothermal flow varies with frequency due to variation in the balance of the Coulomb force and dielectric force. The flow patterns for $\omega=300 \mathrm{kHz}$ and $\omega=1 \mathrm{GHz}$ resemble the flow patterns for low and high frequency limits, where the Coulomb force and dielectric force become dominant, respectively. The flow pattern also strongly depends on the geometry of the system. In the current 3D system, independently of frequency, the electrothermal force gives rise to upward flows on the gap of the electrodes and downward flows on domain boundary $(x=0 \mu \mathrm{m}, y=25 \mu \mathrm{m})$. This differs from the 2D case with an infinitesimal gap, where the direction of the flow circulation at low frequency limit is opposite from that at high frequency limit $12 \mid 13$.

After solving Eq. (4), we obtain the actual trajectories of the s-SWNTs as plotted in Fig. 4(c) and Fig. 4(d), The simulation times are $33 \mathrm{~ms}$ and $24 \mathrm{~ms}$ respectively. The s-SWNTs are seen to follow the streamlines of the electrothermal flow, especially well in the region close to the electrodes. This agrees with the local force comparisons shown in Fig. 3, where the electrothermal force appeared to be dominant in a broad region close to the electrode. This means that the variation of the flow patterns observed in Fig. 4(a) and Fig. 4(b) results in different spatial trapping distributions of SWNTs. When $\omega=300$ $\mathrm{kHz}, \mathrm{s}-\mathrm{SWNTs}$ are attracted broadly on the electrode, while when $\omega=1 \mathrm{GHz}$, the s-SWNTs are mainly attracted to a relatively small region close to the domain boundary.

Let us briefly discuss the influence of the electrothermal effect on m-SWNTs. We have also performed the simulations for m-SWNTs with $\sigma_{p}=10^{8} \mathrm{~S} / \mathrm{m}$ and $\epsilon_{p}=$ $-10^{4} \epsilon_{0}{ }^{11}$. The magnitude of the positive DEP force for $\mathrm{m}-\mathrm{SWNT}$ is much larger than that for s-SWNTs. Consequently, the impact of both electrothermal and Brownian forces on the overall transport is much smaller for $\mathrm{m}$ SWNTs than for s-SWNTs. However, the electrothermal force is far from negligible, especially for higher SDBS concentrations. In fact, in the gap region of the electrode pair where the electrothermal force is directed upwards ( $y=0 \mu \mathrm{m}$ to $5 \mu \mathrm{m}$ in Fig. 4), the electrothermal force overcomes the DEP force and repels m-SWNTs away from the

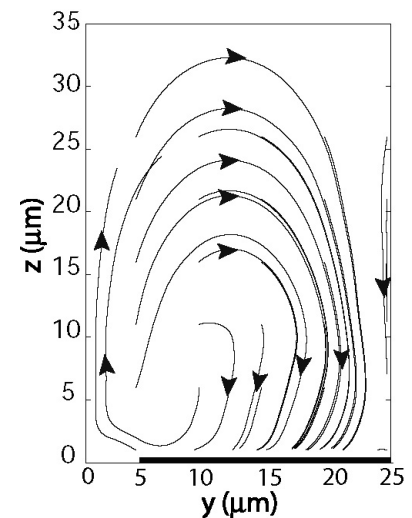

(a)Streamlines of the electrothermal flow, $\omega=300 \mathrm{kHz}$

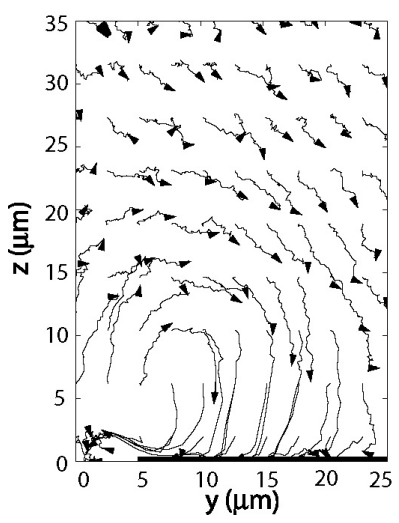

(c) Traces of s-SWNTs, $\omega=300 \mathrm{kHz}$

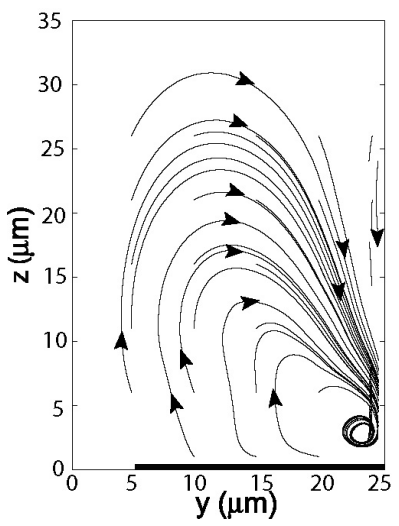

(b) Streamlines of the electrothermal flow, $\omega=1 \mathrm{GHz}$

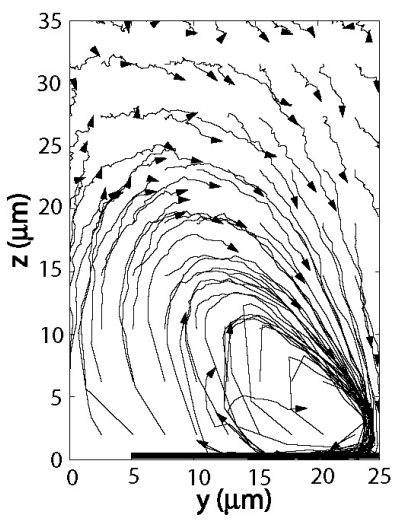

(d)Traces of s-SWNTs, $\omega=1 \mathrm{GHz}$
FIG. 4: Streamlines and corresponding traces of s-SWNTs in $0.1 \%$ SDBS solutions, low $(300 \mathrm{kHz})$ and high $(1 \mathrm{GHz})$ frequency regimes with $\phi=20 \mathrm{~V}$.

electrodes when the concentration of SDBS is larger than $0.1 \%$. The impact on the collection of m-SWNTs may be limited since the m-SWNTs will be circulated along the vortex and eventually transported onto the electrodes after a certain time as seen in Fig. 4 for s-SWNTs. However, the effect should certainly influence the distribution of yielded m-SWNTs on the electrode, which is important for deposition of SWNTs to selected sites for electric device constructions.

\section{CONCLUSIONS}

We formulated a dynamical system to simulate the DEP motion of s-SWNTs, which is different from previous simulations mainly by taking the electrothermal flow into account. To realize the DEP separation, the applied electric potential needs to be high enough so that the DEP force on m-SWNTs overcomes the Brownian motion. This results in a high temperature gradient, which creates a substantial electrothermal flow. 
This electrothermal flow will bring the s-SWNTs close to the electrodes where the weaker DEP force on s-SWNTs can collect them. Thus, the collaborative action of electrothermal flow and DEP restricts the allowable range of potentials where DEP separation may be possible. The higher the concentration of the surfactant is, the stronger the electrothermal flow is. Also, when the frequency increases, the pattern (direction and magnitude) of the electrothermal flow changes. Therefore the electrother- mal flow would very likely increase the difficulty both in separating s-SWNTs from m-SWNTs and in depositing SWNTs to a certain position. The main conclusion of this paper is that when designing DEP separation of SWNTs, it is necessary to consider the possible influence of the electrothermal flow.

The Swedish Research Council (VR) is greatly acknowledged for financial support. The authors thank Z.-B. Zhang and S.-L. Zhang for stimulating discussions.
* Electronic address: gustava@mech.kth.se

1 R. Saito, G. Dresselhaus, and M. S. Dresselhaus, Physical Properties of Carbon Nanotube (Imperial College, London, 1998).

2 R. Krupke, F. Hennrick, H. V. Löhneysen, and M. M. Kappes, Science 301, 344 (2003).

3 Z. Chen, X. Du, M.-H. Du, H.-P. C. C. D. Ranchen, and A. G. Rinzler, Nano Lett. 3, 1245 (2003).

4 D. Chattopadhyay, L. Galeska, and F. Papadimitrakopoulos, J. Am. Chem. Soc. 125, 3370 (2003).

5 M. Zheng, A. Jagota, M. S. Strano, A. P. Santos, P. Barone, S. G. Chou, B. A. Diner, M. S. Dresselhaus, P. S. McLean, G. B. Onoa, et al., Science 302, 1545 (2003).

6 Y. Maeda, S. Kimura, M. Kanda, Y. Hirashima, T. Hasegawa, T. Wakahara, Y. Lian, T. Nakahodo, T. Tsuchiya, T. Akasaka, et al., J. Am. Chem. Soc. 127, 10287 (2005).
7 R. Krupke, F. Hennrick, M. M. Kappes, and H. V. Löhneysen, Nano Lett. 4, 1395 (2004).

8 Z.-B. Zhang, X.-J. Liu, S.-L. Zhang, and E. E. B. Campbell, J. Appl. Phys. 98, 056103 (2005).

9 S. Baik, M. Usrey, L. Rotkina, and M. Strano, J. Phys. Chem. B 108, 15560 (2004).

10 H.-J. Dai, Surface Science 500, 218 (2002).

11 M. Dimaki and P. Bøggild, Nanotechnology 15, 1095 (2004).

12 H. Morgan and N. G. Green, AC Electrokinetics: Colloids and Nanoparticles (Research Studies, England, 2001).

13 A. Ramos, H. Morgan, N. G. Green, and A. Castellanos, Phys. D: Appl. Phys. p. 2339 (1998).

14 G. Amberg, R. Tönhardt, and C. Winkler, Math. Comp. Simulation 49 (1999). 